\title{
LA INSPECCIÓN DE EDUCACIÓN, UN FUTURO INCIERTO
}

\section{The Educational Inspection, an uncertain future}

\author{
Avelino Sarasúa Ortega \\ Inspector de Educación de la Comunidad de Madrid \\ Correo-e: Avelino.sarasua@madrid.org \\ Recibido: I5 de septiembre de 2018 \\ Envío a informantes: 22 de septiembre de 2018 \\ Aceptación definitiva: 3 de noviembre de 2018
}

Resumen: La Inspección Educativa siempre ha sido una referencia para los centros educativos y para el profesorado. Ha sido considerada como un importante factor para la mejora del sistema educativo y un elemento clave para la calidad de la enseñanza. Sin embargo, la incidencia y la relevancia de la inspección son cada vez menores tanto para la Administración educativa como para los propios centros. La Inspección Educativa es una institución que tiene en estos momentos una grave crisis de identidad.

El artículo tiene dos partes bien diferenciadas. En la primera, se hace un breve análisis del momento actual de la Inspección, haciendo referencia a su origen y a su breve historia. Se tratan temas como funciones, atribuciones, dependencia orgánica y funcional y el requisito y forma de acceso. En la segunda se plantean algunos de los retos que tiene que afrontar.

Palabras Clave: Inspección Educativa; funciones y atribuciones; la profesión de la inspección; independencia; autonomía.

Aвstract: The Educational Inspection has always been a reference for educational centers and for teachers. It has been considered as an important factor for the improvement of the educational system and a key element for the quality of education. However, the incidence and relevance of the inspection is decreasing for both the educational administration and the centers themselves. The Educational Inspection is an institution that at the moment has a serious identity crisis.

The article has two distinct parts. In the first one, a brief analysis of the current moment of the Inspection is made, making reference to its origin and its brief history. Topics such as functions, attributions, organic and functional dependency and the 
requirement and form of access are treated. In the second, some of the challenges you have to face are plated.

KeY wORDs: Educational Inspection; functions and attributions; the profession of inspection; independence; autonomy.

\section{Breve análisis de la Inspección de Educación}

\lceil

A INSPECCIÓN ES UN ELEMENTO DEL SISTEMA EDUCATIVo que siempre ha cumplido las funciones que le han sido encomendadas. Ha sido y es un cuerpo muy profesional, técnico y con una gran disciplina.

La Inspección Educativa ha sido siempre considerada como un importante factor de la mejora del sistema educativo y un elemento clave para la calidad de la enseñanza.

Los inspectores de educación siempre han sido una referencia para los centros y para el profesorado. Pienso, sin embargo, que la incidencia de la Inspección es cada vez menor tanto para la Administración como para los centros.

La Inspección de Educación es una institución que tiene en estos momentos una grave crisis de identidad, de no saber realmente cuál es su misión o su papel en el sistema educativo.

La Inspección Educativa siempre ha padecido crisis de identidad: entre el control y el asesoramiento, entre la profesionalización y la politización, entre funciones administrativas y funciones pedagógicas. En estos tiempos nuevos ¿qué funciones debe cumplir un inspector/a? ¿Son necesarios o prescindibles? ¿Deben ser meros agentes de la Administración o garantes de los derechos educativos de todos los ciudadanos?

Alfredo Mayorga (2000) señala que la vida de la inspección continúa debatiéndose con una grave crisis de identidad marcada por el oscilar de la interinidad a la indefinición, que significa, sin duda, los giros copernicanos que han existido. Son numerosas las normas que han regulado de forma diferente la Inspección de Educación: las funciones, el acceso, el carácter docente. Destaca que en el año I988 se modifica la Ley de la Reforma de la Función Pública de 1984 por medio de la Ley 23/1988 de 28 de julio, que crea la función inspectora suprimiendo el cuerpo de Inspección. Posteriormente, la Ley Orgánica 9/1995, de 20 de noviembre (LOPEG), crea la Inspección, como cuerpo docente.

Tomás Secadura plantea como una de sus debilidades la indefinición del papel institucional de la Inspección Educativa, y que en la práctica está perdiendo contenido y relevancia (20II).

La Inspección ha ido perdiendo autoridad, ha perdido una gran parte de su poder y su capacidad de influencia en los centros y en el profesorado. Me atrevo a decir que la Inspección educativa está perdiendo en parte la credibilidad que tenía para la comunidad educativa.

Esta crisis de identidad es debida, a mi entender, a diversos factores. Para analizar estas crisis de identidad me parece muy adecuado y oportuno analizar el origen y la razón de ser de la Inspección; es conveniente valorar la evolución y cambio de las funciones o competencias de la Inspección; ninguna de las actuales funciones de la Inspección son especificas de la Inspección; la dependencia orgánica y funcional de la Inspección Educativa dificulta en gran manera que los inspectores de educación desarrollen su propia labor; se debe hacer una valoración de los requisitos y formación específica necesaria para el acceso a la Inspección. 


\section{Origen y razón de ser de la Inspección}

La Inspección Educativa ha sido siempre una función importantísima y privativa del Estado y tiene como objetivo fundamental y razón de su existir no solo «el cumplir y hacer cumplir las leyes», sino garantizar el derecho a la educación y que dicha educación sea de calidad.

El origen de la Inspección está unido, por lo tanto, a los poderes públicos, ya que de ellos es la competencia. La Inspección recibe su labor por delegación de quien tiene poder y competencia para ejercerla. Es asimismo medio de orientación y ayuda a la realización de una política educativa que tiene su origen en un mandato del Estado y de las correspondientes administraciones educativas.

La Constitución de 1978 establece en el punto 8 del artículo 27 que los poderes públicos inspeccionarán y homologarán el sistema educativo para garantizar el cumplimiento de las leyes.

La Constitución garantiza una Inspección Educativa como una función pública, establece que los poderes públicos organicen y ejerzan la función de inspección sobre el funcionamiento del sistema educativo.

Plantea para la Inspección Educativa una doble misión. Por una parte, supervisar el sistema educativo y, por otra, homologar el sistema educativo. Y ello origina una doble Inspección de Educación: las Inspecciones educativas de cada comunidad autónoma y la alta Inspección dependiente del Estado.

La Inspección Educativa nace en España con carácter profesional referenciada siempre a la enseñanza primaria, obligatoria y gratuita, a mediados del siglo XIX, con la promulgación del Real Decreto de 30 de marzo de i849.

En el Preámbulo del citado Real Decreto se especifica

Tengo el honor de someter a la aprobación de V. M. el crear otra institución hace tiempo reclamada y, sin la cual en vano se afanaría el Gobierno para promover mejoras, pendiendo en gran parte el fruto de sus desvelos y sacrificios. Esta institución es la de los inspectores.

$\mathrm{Si}$ en todos los ramos del servicio público es conveniente esta clase de funcionarios, en la instrucción primaria es indispensable. Sin ellos la administración nada ve, nada sabe, nada puede remediar. Las autoridades no tienen tiempo para vigilar por sí solas tan gran número de establecimientos, ni menos para entrar en la infinidad de pormenores que esta vigilancia exige. Carecen además de los conocimientos especiales que se necesitan para observar muchas cosas que sólo se descubren a los ojos de personas facultativas y amaestradas en esta clase de indagaciones. La creación de los inspectores que han pedido la mayor parte de las provincias, dará la vida a la instrucción primaria y será uno de los medios que más contribuyan a mejorar la educación del pueblo.

Dos finalidades expresan justificadamente la existencia de la Inspección, por un lado, la necesidad de toda Administración de conocer de cerca, de primera mano, lo que ocurre en el ámbito que administra. Por otro, que ese conocimiento sea cualificado, es decir, que sea obtenido y aportado por personas expertas, que puedan interpretar la realidad observada.

Uno de los puntos clave e importantes, que está presente a lo largo de toda la historia de la Inspección, constituye sin duda alguna la manera y modo de delegar los poderes públicos la función inspectora que les corresponde, así como en quién delegan y si confieren a dicha necesidad un carácter objetivo y profesional o lo someten a los imperativos subjetivos de la discrecionalidad y del control político. 
Siglo y medio de historia confiere solera y prestigio a una Institución, la Inspección Educativa, cuyo futuro tiene que pasar necesariamente por estos principios o ejes vertebradores de la profesionalidad, la independencia y la autonomía. Y estos tres principios son claves y esenciales para que la Inspección desarrolle su labor.

El artículo 30 del Real Decreto de 30 de marzo de i849 establece que «las plazas de inspectores provinciales que sacaren en adelante, se proveerán siempre por oposición» y añade que "Considera necesario dar continuidad y permanencia a los inspectores en el ejercicio de su función como medio obligado para garantizar su independencia y su profesionalidad».

Alfredo Mayorga (2000) incide en la independencia de la inspección profesional y resalta que fue suprimida la jurisdicción que sobre ella tenían las autoridades provinciales, y ello supuso como escribió en 1935 Antonio Ballesteros, inspector general de primera enseñanza, «una liberación de la escuela primaria».

La Inspección Educativa nació respondiendo a una necesidad, dicha necesidad no solo sigue existiendo, sino que adquiere cada día mayor importancia y urgencia, y cara al futuro habrá que profundizar en principios y ejes vertebradores que no son otros que la profesionalización, la independencia y la autonomía.

Ha habido momentos históricos en los que esta delegación ha permitido que los inspectores hayan desarrollado su labor con independencia y autonomía. En otros momentos las actuaciones de la inspección han estado sometidas a los imperativos subjetivos de la discrecionalidad y del control político.

La autonomía profesional es una de las preocupaciones más extendida entre todos los inspectores de educación. La autonomía del inspector en su trabajo garantiza su profesionalidad y su no dependencia del poder político.

Antonio Iniesta y otros inspectores (1999) señalan que

a lo largo de su historia, la Inspección se ha visto afectada por las profundas modificaciones producidas en el régimen político del país -Monarquía, República, Dictadura, retornos sucesivos a la Monarquía-. Sus comportamientos tienen que quedar siempre al margen del signo político o ideológico de la Administración, del Gobierno. Desde este punto de vista, hay que reconocer el carácter subordinado de Servidora de la Administración que define a la Inspección. Y es Servidora de la Administración en cada momento histórico, es decir, de la que en cada ocasión ostente legítimamente el poder. De ahí, que aceche a la Inspección el grave peligro de convertirse -o que pretendan convertirla- en instrumento de apoyo político para quien ostente el poder. De ahí, también, la preocupación y la reclamación constante de los Inspectores por disfrutar de la estabilidad profesional que respalde y garantice una actuación independiente, al margen de todo tinte político. En ese ámbito ha de enmarcarse el afán patentizado en los últimos años por los Inspectores, por estar encuadrados en un Cuerpo de profesionales que sea el paraguas protector contra la tentación de exigir adhesiones que pudieran surgir por parte del Poder. Que una cosa es el acatamiento y la aplicación, en su caso, de las órdenes dictadas por quien tiene competencia para ello, y otra muy distinta el colaboracionismo con una determinada línea ideológica.

Carlos Utrera, presidente de la federación ADIDE, a la que pertenecen el $60 \%$ de los inspectores, en su comparecencia en el Congreso de los Diputados el I7 de junio de 2017 denunciaba que «la Inspección no cuenta con la independencia suficiente del poder político para llevar a cabo sus funciones. Con frecuencia se tiende a instrumentalizar la Inspección Educativa como si fuese la guardia pretoriana de la Administración». 
En la Resolución de la Viceconsejera de Educación del País Vasco de is de septiembre de 2017 por la que se aprueba el Plan General trienal de la Inspección de Educación para el trienio 20I7-2020, en el apartado de la visión, se recoge que «la Inspección aspira a actuar profesional y éticamente tanto en las intervenciones en los centros como ante la administración educativa con criterios técnicos e independencia de presiones externas» y en el apartado de VALOREs, se especifica que «el elemento clave que sirve como referencia de los comportamientos y actitudes del personal de la Inspección de Educación es la profesionalidad, sustentada en los siguientes valores entre los que se recogen, la honestidad, responsabilidad, lealtad, respeto y empatía y la Autonomía e independencia de criterio con espíritu crítico y autocrítico».

\section{Evolución de las competencias de la Inspección de Educación}

Es muy instructivo para comprender la evolución histórica de la Inspección de Educación analizar las funciones asignadas a lo largo de la historia. Se han escogido cuatro momentos diferentes: el Real Decreto de 20 de mayo de i849 por el que se aprueba el reglamento de la Inspección, desarrollando así el Real Decreto de 20 de marzo de i849, por el que se crea la inspección profesional; el Decreto de 2 de diciembre de 1932, de la Organización de la Inspección de Educación; la Ley I4/1970, de 4 de agosto, General de Educación, y la Ley Orgánica, de 3 de mayo, de Educación (LOE) (última Ley Orgánica que establece las funciones de la Inspección).

\begin{tabular}{|c|c|c|c|c|c|c|}
\hline \multicolumn{2}{|r|}{$\begin{array}{l}\text { Real Decreto de } 20 \text { de mayo } \\
\text { de I } 849 \text { (artículo I8) }\end{array}$} & \multicolumn{2}{|r|}{$\begin{array}{l}\text { Decreto de } 2 \text { de diciembre } \\
\text { de } 1932 \text { (artículo 17) }\end{array}$} & \multicolumn{2}{|r|}{$\begin{array}{l}\text { Ley General de } 1970 \\
\text { (artículo I42) }\end{array}$} & $\begin{array}{c}\text { LOE } \\
\text { (artículo I5I) }\end{array}$ \\
\hline $4 .^{\circ}$ & $\begin{array}{l}\text { Indagar las necesidades de la } \\
\text { instrucción primaria en sus } \\
\text { respectivas provincias, a fin de } \\
\text { proponer a las autoridades, a } \\
\text { las comisiones provinciales y al } \\
\text { Gobierno cuantas mejoras crean } \\
\text { convenientes para el aumento y } \\
\text { prosperidad de las escuelas. } \\
\text { Vigilar sobre el cumplimiento } \\
\text { de las leyes, decretos, reglamen- } \\
\text { tos y demás disposiciones vi- } \\
\text { gentes relativas a la Instrucción } \\
\text { Primaria, excitando el celo de } \\
\text { las autoridades, denunciando } \\
\text { las faltas y abusos, y haciendo } \\
\text { a quien corresponda las oportu- } \\
\text { nas reclamaciones. } \\
\text { Gestionar para que los maestros } \\
\text { estén puntualmente pagados, y } \\
\text { se les trate con el decoro debido. } \\
\text { Investigar los fondos con que se } \\
\text { sostienen las escuelas, y vigilar } \\
\text { sobre la recta administración de } \\
\text { los bienes que estén aplicados, } \\
\text { sobre el cumplimiento de las cláu- } \\
\text { sulas de las fundaciones, y sobrela } \\
\text { puntual rendición de cuentas por } \\
\text { las que están obligados a darlas, } \\
\text { dirigiendo para todo ello sus re- } \\
\text { clamaciones a quien corresponda. }\end{array}$ & I. $^{\circ}$ & $\begin{array}{l}\text { Orientar, impulsar y dirigir } \\
\text { las Escuelas nacionales y las } \\
\text { Instituciones auxiliares de las } \\
\text { mismas. Contribuir al mejo- } \\
\text { ramiento profesional de los } \\
\text { Maestros. Perfeccionar la vida } \\
\text { pedagógica de las Escuelas e } \\
\text { intensificar su acción social. } \\
\text { Cuidar especialmente de que } \\
\text { sea respetada en todo mo- } \\
\text { mento la conciencia del niño, } \\
\text { garantizando el más escrupu- } \\
\text { loso cumplimiento de laicis- } \\
\text { mo y de las Leyes que ampa- } \\
\text { ran los derechos e intereses de } \\
\text { la infancia. } \\
\text { Visitar las demás Escuelas } \\
\text { públicas y privadas para com- } \\
\text { probar si en su labor se some- } \\
\text { ten a los preceptos legales y a } \\
\text { las condiciones en que fue au- } \\
\text { torizado su funcionamiento. } \\
\text { Conceder a los Maestros de su } \\
\text { zona votos de gracias y propo- } \\
\text { nerles para otras recompensas, } \\
\text { como asimismo imponerles } \\
\text { la sanción de apercibimiento } \\
\text { o proponer la aplicación de } \\
\text { otras penas, previa formación } \\
\text { de expedientes gubernativos. }\end{array}$ & b) & $\begin{array}{l}\text { Velar por el cumplimiento } \\
\text { de las leyes, Reglamentos y } \\
\text { demás disposiciones en todos } \\
\text { los centros docentes estatales } \\
\text { y no estatales, en el ámbito de } \\
\text { la función educativa. } \\
\text { Colaborar con los Servicios } \\
\text { de Planeamiento en el estu- } \\
\text { dio de las necesidades educa- } \\
\text { tivas y en la elaboración y ac- } \\
\text { tualización del mapa escolar } \\
\text { de las zonas donde ejerza su } \\
\text { función, así como ejecutar in- } \\
\text { vestigaciones concernientes a } \\
\text { los problemas educativos de } \\
\text { éstas. } \\
\text { Asesorar a los profesores de } \\
\text { los centros estatales y no es- } \\
\text { tatales sobre los métodos más } \\
\text { idóneos para la eficacia de la } \\
\text { enseñanza que imparten. } \\
\text { Evaluar el rendimiento edu- } \\
\text { cativo de los centros docen- } \\
\text { tes y profesores de su zona } \\
\text { respectiva o de la especiali- } \\
\text { dad de su cargo en colabo- } \\
\text { ración con los Institutos de } \\
\text { Ciencias de la Educación. }\end{array}$ & $\begin{array}{l}\text { a) Supervisar y controlar, desde } \\
\text { el punto de vista pedagógico y } \\
\text { organizativo, el funcionamien- } \\
\text { to de los centros educativos así } \\
\text { como los programas que en } \\
\text { ellos inciden. } \\
\text { b) Supervisar la práctica docente, } \\
\text { la función directiva y colaborar } \\
\text { en su mejora continua. } \\
\text { c) Participar en la evaluación del } \\
\text { sistema educativo, de los ele- } \\
\text { mentos que lo integran. } \\
\text { d) Velar por el cumplimiento, en } \\
\text { los centros educativos, de las } \\
\text { leyes, reglamentos y demás dis- } \\
\text { posiciones vigentes que afecten } \\
\text { al sistema educativo. } \\
\text { e) Velar por el cumplimiento y } \\
\text { aplicación de los principios } \\
\text { y valores recogidos en esta } \\
\text { Ley, incluidos los destinados a } \\
\text { fomentar la igualdad real entre } \\
\text { hombres y mujeres. } \\
\text { f) Asesorar, orientar e informar a } \\
\text { los distintos sectores de la co- } \\
\text { munidad educativa en el ejerci- } \\
\text { cio de sus derechos y en el cum- } \\
\text { plimiento de sus obligaciones. }\end{array}$ \\
\hline
\end{tabular}




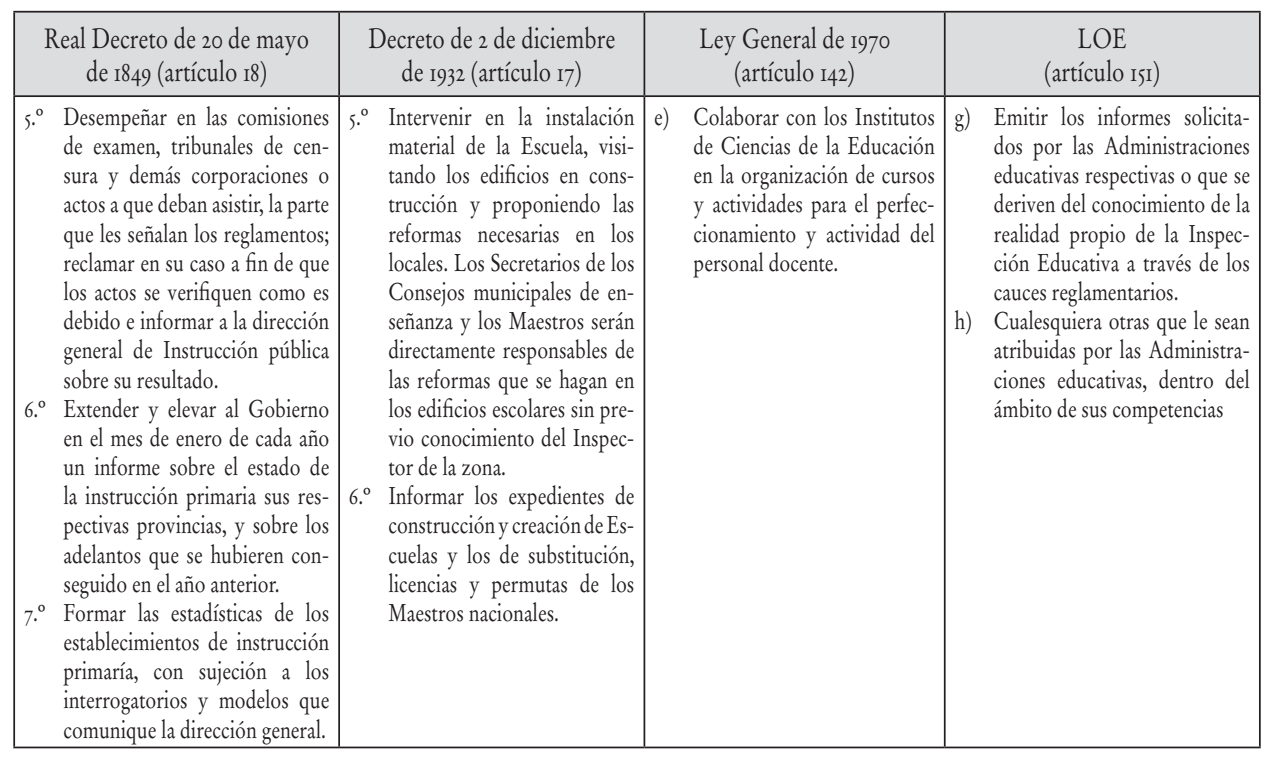

El análisis comparativo de las funciones asignadas a la Inspección educativa en diferentes y distintas épocas pone de manifiesto que existe una gran diferencia tanto en la naturaleza de las funciones de la inspección como las consecuencias de sus actuaciones. Se exponen a continuación algunas valoraciones:

r. ${ }^{a}$ En un análisis comparativo de las funciones iniciales de la Inspección con las establecidas actualmente en la LOE, se observa que solo dos funciones coinciden «Vigilar o velar por el cumplimiento de la norma» y «Elevar informes». Las competencias actuales de la inspección son muy diferentes y distintas a sus funciones o responsabilidades iniciales.

2. ${ }^{a}$ En el Real Decreto de 20 de mayo de 1849 tienen una gran importancia las funciones que deben llevar a cabo los inspectores de educación en relación con la planificación y gestión educativas. Sin embargo, en el preámbulo del Decreto de 2 de diciembre de 1932, por el contrario, se recoge y justifica que ha llegado el momento de sistematizar lo que ha hecho en este sentido la República y completarlo de suerte que quede destacada la nueva fisonomía de la función inspectora. Las normas en que se inspira son: liberarla de preocupaciones burocráticas intensificando su carácter técnico; acercar el inspector a la Escuela y al maestro con afán tutelar, con ánimo de aportar su ciencia y su experiencia para infundir nuevo espíritu a la enseñanza; convertirle en profesor ambulante, transformándole, por tanto, en verdadero consejero escolar que trabaja en la Escuela con el maestro, y como maestro ofreciendo el ejemplo de sus lecciones modelo.

Son dos planteamientos diferentes de la Inspección. En uno prima el velar por el cumplimiento de la norma y la supervisión, en el otro el asesoramiento y la ayuda al profesorado.

$3 \cdot{ }^{a}$ Los informes de los inspectores en su inicio eran destinados o dirigidos al propio Gobierno. La actuación del inspector tenía una gran incidencia y repercusión social no solo en los centros, sino también en los ayuntamientos y en los poderes políticos. 
María Teresa López del Castillo (1999) señala que, en los primeros años de creación del cuerpo, la Revista de Instrucción Primaria informa ampliamente de los logros conseguidos mediante las visitas de los inspectores. Sirva como ejemplo el escrito remitido por los profesores del partido de Béjar elogiando la actuación del inspector don Cesáreo Antolín Viñe, que en sus visitas a los pueblos del partido ha conseguido la reforma de algunos locales antiguos y la construcción de varios edificios nuevos para las escuelas; la dotación de material y de los enseres necesarios; la mejora en la disciplina y en los métodos de enseñanza; la animación de las comisiones locales, interesando en favor de la educación primaria a los ayuntamientos y a los padres de familia; el pago de los atrasos a varios maestros y el aumento de las dotaciones a otros. Estas serán las cuestiones que solían abordar los inspectores, tal como vemos en muchas informaciones semejantes en la misma revista.

Los informes actuales de la Inspección no tienen generalmente ninguna o escasa incidencia social. Me atrevo a decir incluso que tienen una incidencia escasa para la propia Administración educativa.

Nunca o casi nunca los informes de Inspección son públicos y únicamente son conocidos por la propia Administración educativa. Los informes de inspección, por otra parte, no tienen nunca un carácter vinculante.

Este aspecto va a ser modificado por la Ley 39/2015, de i de octubre, del Procedimiento Administrativo Común de las Administraciones Públicas, en la que según el artículo 70 los informes de inspección deberán formar parte de los posibles Expedientes Administrativos que tendrán siempre un carácter electrónico.

$4{ }^{a}$ Las funciones de la Inspección educativa establecidos en el Decreto de la República nos permiten observar un nuevo modelo y un nuevo planteamiento de la Inspección. La función de asesoramiento y de orientación que está en todas las disposiciones legales, excepto en el Real Decreto inicial de la Inspección, es la función clave y esencial en el Decreto de la República.

$5{ }^{a}$ Una parte importante de las funciones asignadas en su origen a la Inspección de educación han ido sido asumidas de forma progresiva por nuevas unidades administrativas. La creación de las Consejerías de Educación y el crecimiento y aumento de la estructura de las delegaciones o direcciones provinciales ha permitido que la planificación educativa y la gestión de los centros y programas estén más próximas a los propios centros educativos. Los directores y directoras escolares mantienen una relación directa con la propia Administración. Los inspectores de educación no tienen ya responsabilidad de fijar las unidades y grupos ni establecer el cupo y la plantilla que debe tener un centro. Los directores y directoras no tienen que acudir a «su inspector» para conseguir una unidad o un profesor más. El posible informe de Inspección en este aspecto, no siempre es tenido en cuenta.

6. ${ }^{a}$ La formación del profesorado ha estado tradicionalmente ligada a la labor de la Inspección Educativa.

El artículo 20 del Real Decreto de 30 de marzo de 1849 establece que los inspectores en las provincias donde exista una escuela normal elemental tendrán la obligación de enseñar en ella en ciertas épocas de año las materias que se les señale. El Decreto de 2 de diciembre de la organización de la Inspección recoge como funciones la de contribuir al mejoramiento profesional de los maestros y perfeccionar la vida pedagógica de las escuelas. 
La Ley 70 recoge entre las funciones de la Inspección «Colaborar con los Institutos de Ciencias de la Educación en la organización de cursos y actividades para el perfeccionamiento y actividad del personal docente».

Me parece oportuno comentar y analizar a creación de los CEIREs (los círculos de estudios e intercambios para la renovación pedagógica), regulados por la Orden de 3 de agosto de 1983 y antecesores de los centros de profesores.

Los CeIRes se constituyen como agrupación de maestros tanto de centros públicos como privados para el perfeccionamiento, intercambio de experiencias y actualización del ejercicio de su función docente.

Los CEIREs dependían del director provincial, quien, por medio de la Inspección de Educación, ejercía las funciones de estimulación, asesoramiento, coordinación general, evaluación de resultados y control económico de los mismos.

Sin embargo en las leyes orgánicas posteriores no existe ningún tipo de relación entre formación del profesorado y la Inspección Educativa.

$7 \cdot{ }^{a}$ Una valoración de las funciones asignadas a la Inspección Educativa en las diferentes leyes orgánicas permite comprobar que cada vez la Inspección tiene menos funciones que le son propias y específicas.

Un análisis comparativo de las funciones de la Inspección Educativa con las competencias del director, del Consejo Escolar y del Claustro de profesores muestra que ninguna de las funciones de la Inspección es una competencia específica y exclusiva de la Inspección.

El artículo I32.d de la LOE, modificado por la LOMCE, que regula las competencias del director, establece entre sus funciones garantizar el cumplimiento de las leyes y demás disposiciones vigentes y el artículo i5I.d) de la LOE recoge entre las funciones de la Inspección Velar por el cumplimiento, en los centros educativos, de las leyes, reglamentos y demás disposiciones vigentes que afecten al sistema educativo.

El artículo I5ı.c) establece entre las funciones de la Inspección la de participar en la evaluación del sistema educativo y de los elementos que lo integran. El artículo I27.a) de la LOE, modificado por la LOMCE, que regula las competencias del Consejo Escolar, recoge en su apartado a) «Evaluar los proyectos y las normas a los que se refiere el capítulo II del título v de la presente Ley orgánica».

El artículo I29 de la LOE, que establece las competencias del claustro de profesores, establece en su apartado a) «Aprobar y evaluar la concreción del currículo y todos los aspectos educativos de los proyectos y de la programación general anual».

\section{Escasas atribuciones de la Inspección para desarrollar su labor}

El artículo I53 de la LOE establece que para cumplir las funciones de la Inspección Educativa los inspectores tendrán una serie de atribuciones.

Los reglamentos que regulan las diferentes inspecciones educativas en las CC. AA. no tienen un planteamiento homogéneo en relación con las atribuciones de los inspectores.

Se ofrece un breve resumen de las atribuciones que se recogen en algunos de los reglamentos de la Inspección:

- Visitar los centros docentes (públicos y privados), servicios y programas.

- Tener acceso y supervisar la documentación académica y administrativa de los centros. 
- Convocar, celebrar y presidir reuniones con los miembros de los diferentes órganos de gobierno y de coordinación docente de los centros, así como con los de los diferentes sectores de la comunidad educativa.

- Elevar informes y levantar actas, cuando proceda, por propia iniciativa o a instancias de las autoridades de la Administración educativa.

- Requerir, a través de los cauces establecidos, a los responsables de los centros docentes, servicios y programas para que adecuen su organización y funcionamiento a la normativa vigente.

- Intervenir en los procedimientos disciplinarios que se les asignen.

Si entendemos por atribución la asignación de unas tareas a unos profesionales para que puedan desarrollar su labor, se pueden considerar estas atribuciones como instrumentos o recursos que los inspectores tienen para llevar a cabo su labor.

Pero si entendemos por atribuciones las facultades que el cargo o la tarea que ejerce le dan a un profesional, se puede concluir que el inspector de educación tiene pocas atribuciones, poca autoridad o poco poder para cumplir con sus funciones.

Visitar un centro, supervisar una documentación, mantener una reunión son atribuciones que son más bien instrumentos para el trabajo del inspector. ¿Pero realmente qué capacidad tiene un inspector para exigir el cumplimiento de una norma? ¿ 'Tiene las mismas atribuciones que un inspector del Ministerio de Hacienda o del Ministerio de trabajo? ¿Sus actuaciones tienen los mismos efectos o consecuencias?

Los inspectores de educación son autoridad, pero no disponen del poder para llevar a cabo su labor inspectora.

\section{La dependencia de la Inspección de Educación}

La Inspección de educación tiene una doble dependencia, la dependencia orgánica y la dependencia funcional. Este siempre ha sido un tema controvertido. La Inspección de educación depende funcionalmente de una estructura central de la Administración, que establece los planes de actuación de la Inspección Educativa, pero orgánicamente depende del delegado o director provincial de Educación. Actualmente, una gran parte de las actuaciones de los planes de actuación de la Inspección, no se puede realizar no tanto por la gran cantidad de tareas y actividades propuestas y por número de centros y programas (es una realidad), sino sobre todo por las tareas que se le asignan que no son propias y específicas de la Inspección.

\section{La Inspección de Educación, el final de la carrera docente}

El cuerpo de Inspección, creado por la LOPEG como cuerpo docente, es considerado por muchos como el final de la carrera docente, y no como una profesión diferente a la docencia y diferente a la dirección. Y, por lo tanto, como en el caso de la dirección, no se le exige una formación específica y propia.

Todo ello nos hace volver a que la Inspección está en un momento de crisis de identidad y la necesidad de repensar la razón de ser de la Inspección Educativa. 


\section{Retos que debe afrontar la Inspección de Educación}

La Inspección Educativa debe dar vida a los centros y debe ser uno de los medios que más contribuyan a mejorar la Educación. De lo contario la Inspección Educativa, como ya ocurre en otros países (ejemplo de Finlandia), no tendrá razón de ser y puede estar llamada a desaparecer. No tiene ningún sentido tener a funcionarios altamente cualificados llevando a cabo muchas veces meras tareas administrativas y burocráticas. La labor de la Inspección tiene que incidir significativamente en el aula, en la mejora de los procesos de enseñanza y aprendizaje y en la mejora de la organización y funcionamiento de los centros.

Es necesario repensar la Inspección Educativa, es necesario establecer la finalidad y su razón de ser y enderezar el rumbo de sus acciones.

Estos son algunos de los posibles retos:

\section{I. ${ }^{\circ}$ Definir con claridad las competencias de la Inspección Educativa}

Es necesario repensar cuál es la misión, el porqué y el para qué de la Inspección Educativa. Es necesario definir con claridad cuáles son sus competencias.

Para ello es necesario mirar hacia adelante, no sería adecuado volver la vista hacia atrás.

Es necesario establecer cuáles son sus funciones o competencias, asumiendo que es un conjunto de profesionales con alto nivel de formación y preparación profesional.

Desde mi punto de vista dos son los grandes ámbitos de la Inspección Educativa, la supervisión y la evaluación.

En el ámbito de la supervisión, la Inspección debe, como se establece en la propia LOE, velar por el cumplimiento, en los centros y programas educativos, de las leyes, reglamentos y demás disposiciones vigentes que afecten al sistema educativo. Debe supervisar que el profesorado desarrolle y lleve a la práctica sus responsabilidades en la labor docente y debe verificar que las direcciones escolares ejerzan las competencias de la función directiva.

A una mayor necesidad de la autonomía de los centros, más necesaria la función de supervisión.

En relación con la evaluación del sistema educativo, la Inspección debe participar y colaborar en los diferentes programas y planes de evaluación.

Dada su situación en el sistema educativo y su cualificación, la Inspección debe responsabilizarse directamente de los planes de la evaluación de la labor docente y de la función directiva y de los planes de evaluación de los centros educativos.

Tanto la evaluación de la práctica docente del profesorado como la evaluación de los centros son asignaturas pendientes de la Administración educativa. Desde la LOGSE todas las leyes orgánicas han establecido como una finalidad esencial la calidad educativa y la mejora del sistema educativo.

No es posible mejorar nada si no se evalúa. Y en este país, únicamente se evalúa al alumnado (evaluaciones internas y diferentes pruebas externas). Nadie duda de que el profesorado y las direcciones de los centros sean factores esenciales que explican en una parte importante los resultados escolares obtenidos por el alumnado. Diferentes informes internacionales concluyen que, tras la acción docente del profesorado, la dirección escolar es el segundo factor interno que tiene mayor incidencia en los logros del aprendizaje y en los resultados escolares. Y, sin embargo, en España los diferentes 
intentos de planes de evaluación de profesorado y de los centros han sido esporádicos $\mathrm{y}$ sin ninguna continuidad. Y hoy estos planes son inexistentes.

\section{2. ${ }^{\circ}$ La Inspección de Educación es una profesión, no es el final de la carrera docente}

Las funciones y obligaciones del profesorado, de la dirección escolar y de la Inspección están establecidas y diferenciadas en la Ley Orgánica 2/2006, de 3 de mayo, de Educación (LOE). La docencia, la dirección y la inspección son tres profesiones diferentes. Sin embargo, a veces son consideradas niveles diferentes de la carrera docente. Y no es así. Son profesiones diferentes y que exigen una formación inicial distinta y diferenciada.

Para asumir la dirección, el profesor debe tener el requisito de unos años de experiencia docente y participar en un concurso de méritos. Para acceder a la Inspección, el profesor debe tener un mínimo de años como docente y aprobar el concurso oposición, en el que se valoran los años en la dirección. No se exige en ningún caso una formación específica. Este es un grave error de nuestro sistema educativo.

Si analizamos las funciones de la Inspección que se recogen en la LOE (supervisar y controlar, supervisar, evaluar, velar por el cumplimiento, asesorar, orientar e informar) se puede comprobar que un profesor por el hecho de preparar un temario de una oposición y de haberla superado no tiene la formación suficiente y no ha adquirido las capacidades necesarias para desarrollar la labor como inspector de educación.

Una vez asignadas las funciones o competencias es necesario definir el perfil competencial de los inspectores de educación y establecer sus competencias profesionales.

Buj Gimeno (1992) plantea que el inspector ha de ser competente, discreto y prudente.

La competencia comporta conocimiento teórico y dominio de técnicas.

La discreción, capacidad de saber distinguir cuestiones y sus aspectos para saber determinar cómo, cuándo y a quién conviene.

La prudencia permite la aplicación correcta de una norma o criterio en una circunstancia concreta.

La capacitación científica del inspector profesional exige un conocimiento teórico y la adquisición de unas técnicas que se suelen llamar competencias conceptuales y competencias técnicas. La discreción y la prudencia son competencias humanas.

Buj Gimeno (1992) hace hincapié en diferenciar ambas competencias, las competencias conceptuales y técnicas y las competencias humanas. Entre estas tampoco puede olvidarse el componente ético.

Esto nos obliga necesariamente a analizar y valorar la forma de acceso a la Inspección de Educación, tanto los requisitos como el propio carácter del concurso oposición.

La oposición puede ser un buen instrumento para valorar las competencias científicas y las competencias técnicas, pero no las competencias humanas.

Tanto el Real Decreto de 30 de marzo de 1849 como el Decreto de 2 de diciembre de i93 I establecían el requisito de una formación previa específica: tres años en la Escuela Normal Central o en cualquier de las escuelas superiores de magisterio o tener el grado de la sección de Pedagogía de la Facultad de Filosofía y Letras, respectivamente.

Se debe hacer notar que son requisitos para acceder a la Inspección de la Instrucción Primaria, no para la Enseñanza Secundaria.

Sin embargo, es necesario resaltar la fuerte formación exigida en las Ciencias de la Educación (Pedagogía, Didáctica) que se exigía para acceder a la Inspección. Y esto es clave. No se puede acceder a la Inspección sin una preparación en las Ciencias de la 
Educación. Un licenciado en Matemáticas, o en Geografía e Historia y con unos años de experiencia docentes no tiene la formación necesaria para ser inspector, aunque haya aprobado la oposición.

Como el máster en educación para acceder a la Enseñanza Secundaria, se debe valorar la necesidad de un máster específico para acceder a la Inspección.

La formación científica tiene una doble vertiente: la formación pedagógica y la formación académica.

Buj Gimeno (I992) especifica que la capacitación científica del inspector profesional exige que este tenga conocimientos en Fundamentos teóricos de la educación, Teoría general de sistemas, Política de la educación, Sociología de la educación, Psicología de la educación, Sistemas educativos comparados, Fundamentos de administración, Organización y planificación educativa, Programación didáctica, Modelos y técnicas de evaluación, Teoría y técnicas de toma de decisiones, Dinámica de grupos, Métodos de investigación y estadística aplicada a la educación.

Los contenidos de estas materias muestran los conocimientos específicos, nos pueden permitir diseñar lo que pudiera ser un plan de estudios para el inspector, que pueden formar parte del máster propuesto.

Si bien la formación académica es la adecuada, ya que todos inspectores provienen de una especialidad y de un cuerpo docente, no todos los inspectores de educación tienen una formación en las Ciencias de la Educación (Pedagogía, Didáctica...), totalmente necesaria para ejercer la profesión. La visita al aula, actuación que ha quedado en muchos casos relegada, puede encontrar aquí uno de los motivos.

Evidentemente, la capacitación en las materias precedentes es compatible con una experiencia docente que, a su vez, comporta la existencia en el inspector de otra faceta, hasta ahora adoptada normalmente como elemento fundamental de su capacitación, a saber, pertenecer al profesorado en alguno de los niveles en que se configura el sistema educativo; dicha condición implica el haber sido formado académicamente en una especialidad concreta de la enseñanza.

\section{3. ${ }^{\circ}$ Acceso a la Inspección}

El acceso a la Inspección Educativa, como a los cuerpos docentes, tiene que exigir unos requisitos previos.

En relación con requisitos previos serían necesarios un mínimo de años de experiencia docente y una formación específica.

El requisito de un mínimo de años de experiencia nadie duda de su necesidad. La condición de pertenecer a un cuerpo docente ha sido siempre un requisito, no así, sin embargo, en la LOCE, que estableció unas oposiciones abiertas, a docentes no funcionarios.

Como ya se ha señalado, si ejercer la Inspección de Educación es una profesión y no es final de la carrera docente, sería necesario no solo acreditar un titulo de una enseñanza superior, sino tener una formación específica, como puede ser un máster en la Inspección Educativa.

Como ya se ha señalado anteriormente el artículo 17 del Real Decreto de 30 de marzo de I849 sobre escuelas normales e inspectores de Instrucción Primaria establecía que para optar al cargo de inspección se necesita haber cursado los tres años en la Escuela Central o en cualquiera de las superiores y el artículo 27 del Decreto de 2 de 
diciembre de 1932, sobre la organización de la Inspección de Educación, fijaba como requisito para acceder a la inspección ser graduado en la Sección de Pedagogía de la Facultad de Filosofía y Letras.

La oposición, como ya se ha señalado, puede valorar las competencias científicas y técnicas.

La valoración de las competencias humanas necesariamente exige un periodo de prácticas (mínimo de uno o dos años), en el que se pueda valorar el desarrollo profesional del candidato a la Inspección.

\section{4. ${ }^{\circ}$ El Estatuto de la Inspección Educativa}

Un cuerpo único estatal, la existencia de inspecciones educativas en cada una de las comunidades autónomas, las disfunciones que plantea la doble dependencia, todo ello aconseja que se elabore un estatuto de la Inspección Educativa fuerte y con prestigio que lleve a cabo las funciones que se le asignen.

La Inspección tiene que alcanzar una autonomía profesional en el desarrollo de sus funciones.

\section{La dependencia de la Inspección de Educación}

Es necesario y urgente que las autoridades provinciales educativas tengan limitada su incidencia en el desarrollo de la labor de los inspectores de educación. Se considera necesario recordar la supresión de la jurisdicción sobre la Inspección (I935) que sobre ella tenían las autoridades provinciales.

Quizás en estos momentos no se pueda suprimir la jurisdicción de las autoridades educativas provinciales sobre la Inspección de Educación, pero sí limitarlo, como ya se ha especificado.

\section{6. ${ }^{\circ}$ Necesidad de que la Inspección de Educación recupere la autoridad y el prestigio}

No es solo un problema de la Inspección, es una necesidad de toda la Administración pública.

La Inspección tiene que recuperar la autoridad, tiene que recuperar el prestigio y la capacidad de influencia en los centros y en la sociedad. Es una tarea que va a costar mucho trabajo y mucho tiempo, y que va a exigir la complicidad de todos los inspectores de educación.

\section{7. ${ }^{\circ}$ Las TIC y el trabajo de Inspección}

Las TIC son una buena y potente herramienta de trabajo. Su utilización hace necesaria una nueva y moderna organización y funcionamiento de la Inspección de Educación.

Las TIC pueden y deben modificar la relación y la comunicación de los centros, con las direcciones y con el profesorado. Las TIC ofrecen nuevas y potentes posibilidades, no son una panacea, pero son un instrumento que los inspectores deben utilizar.

No planteo una inspección on line, pero sí que se debe utilizar como recurso. Las posibles aplicaciones informáticas son un nuevo instrumento de supervisión. 


\section{Bibliografía}

Buj Gimeno, Álvaro (1992) Competencias profesionales de los Inspectores de educación. Inspección técnica, sistema educativo e innovación. Universidad Complutense de Madrid. Dpto. de Teoría de la Educación.

Iniesta, A.; Alhambra, C. y Cirac, V. (1999) La inspección en la educación secundaria. $R e-$ vista de Educación, 320, 39-59.

López del Castillo, M. T. (I999) Un proyecto de ley sobre inspección de la enseñanza en el siglo XIX. Bordón. Revista de Pedagogía, 265-276.

Mayorga, A. (2000) La Inspección Educativa. Siglo y medio de Inspección Educativa en España. Madrid: Santillana.

Secadura Navarro, T. (20iI) Principios de organización de la Inspección Educativa en el estado español: fortalezas y debilidades. Revista Avances en Supervisión Educativa. 